\title{
Malignant Phyllodes Tumor Including Aneurysmal Bone Cyst-Like Areas in Pregnancy - a Case Report and Review of the Literature
}

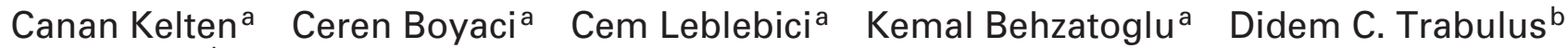 \\ Serkan Sari ${ }^{b}$ Mehmet A. Nazlic Erol R. Bozkurt ${ }^{a}$ \\ a Pathology, Istanbul Research and Training Hospital, Istanbul, Turkey; \\ ${ }^{b}$ General Surgery, Istanbul Research and Training Hospital, Istanbul, Turkey; \\ ${ }^{\mathrm{c}}$ Radiology, Istanbul Research and Training Hospital, Istanbul, Turkey
}

\section{Established Facts}

- The phyllodes tumor is a rare neoplasm that can exacerbate with local recurrence and even metastasis.

\section{Novel Insights}

- The first case of a malignant phyllodes tumor with aneurysmal bone cyst-like areas in a newly delivered woman is reported.

- Since breast masses may mimic benign conditions due to the increased volume of the breast parenchyma during pregnancy and the lactating period, careful breast examination at the beginning of pregnancy is important.

\section{Keywords}

Breast - Phyllodes tumor · Pregnancy .

Aneurysmal bone cysts

\section{Summary}

Background: Malignant phyllodes tumors of the breast are rare biphasic neoplasms. Only few cases related to pregnancy have been reported. Case Report: A 37-yearold woman presented with swelling and pain in her left breast as well as hyperemia on the breast skin, 4 weeks after labor. In her family history, her aunt and maternal cousin had had a breast cancer diagnosis. Clinical evaluation of the patient was consistent with a breast abscess. Therefore, abscess drainage and biopsy from the cavity wall were performed. However, the biopsy was diagnosed as malignant phyllodes tumor. An evaluation by ultrasonography showed a well-defined hypoechoic mass with many cystic spaces covering the entire breast tissue. Therefore, a simple mastectomy was performed. Microscopic examination revealed a high-grade malignant phyllodes tumor. Additionally, bone cyst-like areas in the form of sponge-like blood-filled non-endothelialized spaces were observed. Conclusions: Since the breasts become larger due to the physiological changes during pregnancy, any underlying breast lesions may be obscured. Therefore, clinical breast examination in the first visit of pregnancy is important.

(c) 2016 S. Karger GmbH, Freiburg

\section{KARGER}

() 2016 S. Karger GmbH, Freiburg

Fax +497614520714 


\section{Introduction}

Phyllodes tumors (PTs) are rare neoplasms [1] composed of both epithelial and stromal components. They may occur at any age but mostly in women in the sixth decade. Greater than $70 \%$ of PTs are benign, but borderline and malignant counterparts can be observed [1]. A PT may present during pregnancy like all other breast tumors, but this is very rare. To the best of our knowledge, 8 cases of PT related to pregnancy have been reported in the literature [2-9]. We report another malignant PT in a 37-year-old newly delivered woman. Our case showed common aneurysmal bone cyst $(\mathrm{ABC})$-like areas in the tumor, which is different from the cases reported previously.

\section{Case Report}

A 37-year-old woman presented with swelling and pain in her left breast as well as hyperemia on the breast skin, 4 weeks after labor. She did not have any symptoms during pregnancy. In her family history, her aunt and maternal cousin had had a breast cancer diagnosis. Clinical evaluation of the patient was consistent with a breast abscess. Abscess drainage and biopsy from the cavity wall were performed. However, the biopsy was diagnosed as malignant PT. Ultrasound showed a mildly lobulated, hypoechoic mass $(127 \times 108 \mathrm{~mm})$, with many cystic spaces covering the entire breast tissue and causing prominent asymmetrical distortion. Therefore, a simple mastectomy was performed. Macroscopically, the lesion was $13 \mathrm{~cm}$ in the largest dimension and closer than 1 $\mathrm{mm}$ to the fascia of the pectoral muscle. It was partially well circumscribed, grey-white in color, with many hemorrhagic cystic spaces on the cross-section. There was an ulcerated area $(2.5 \times 2 \mathrm{~cm})$ on the breast skin adjacent to the lesion. 2 lymph nodes were dissected from a small amount of axillary adipose tissue. Microscopically, the tumor was composed of a solid mass of proliferating cells with spindle to epithelioid morphology and focal areas of necrosis. The nuclei of the tumor cells were enlarged; they showed pleomorphism and vesiculation and had conspicuous nucleoli (fig. 1a, b). Increased mitotic activity (10/10 high-power fields (HPF)) including atypical mitoses was noted. The tumor showed invasion into the breast skin. A detailed examination of hematoxylin and eosin (H\&E)-stained slides revealed residual areas of benign fibroepithelial tumor, mostly at the periphery of the tumor. No specific sarcomatoid area such as leiomyosarcoma, liposarcoma, chondrosarcoma, osteosarcoma, or angiosarcoma was observed. Additionally, sponge-like, blood-filled, non-endothelialized spaces (CD31 negative) of various diameters were seen (fig. 1c, d). Some of these spaces were lined with multinucleated osteoclastic giant cells reminiscent of ABC-like areas (fig. 1e). These multinucleated giant cells showed positive staining for CD68, consistent with a histiocytic origin (fig. 1f). The neighboring benign parenchyma of the breast demonstrated common lactational changes. No metastasis was detected in 2 axillary lymph nodes. The malignant tumor cells showed diffuse nuclear positive staining for p53, weak nuclear positive staining for the progesterone receptor $(<10 \%)$, and negative staining for the estrogen receptor.

\section{Discussion}

Phyllodes tumors are biphasic neoplasms that include both an epithelial and a stromal component. They are divided into 3 groups as benign, borderline, and malignant, histopathologically. Most of them show benign features like pushing margins, mild nuclear atypia, and mild stromal cellularity. If the tumor has infiltrative

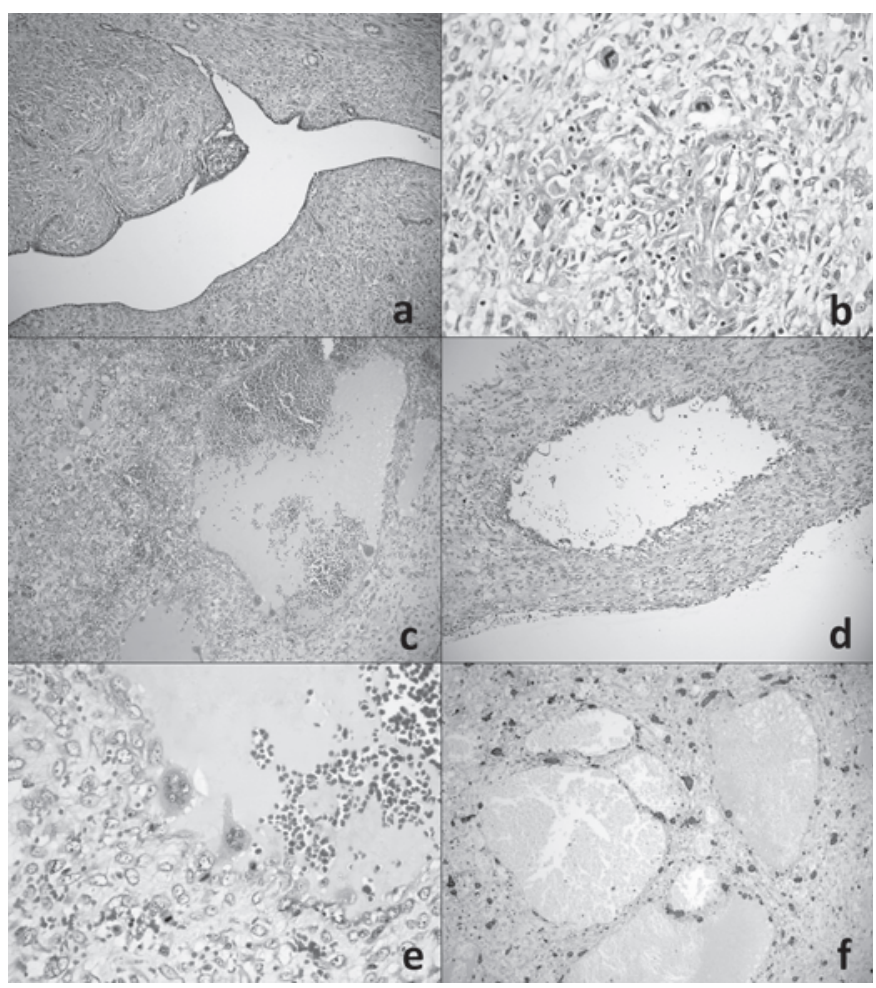

Fig. 1. (a) Solid proliferation of tumor cells forming leaf-like projections $(\mathrm{H} \& \mathrm{E}, \times 100)$. (b) Epithelioid, polygonal and spindle-shaped malignant tumor cells $(\mathrm{H} \& \mathrm{E}, \times 400)$. (c) Blood-filled, anastomosing spaces against a hemorrhagic background $(\mathrm{H} \& \mathrm{E}, \times 100)$. (d) Tumor cells forming septa between cysts $(\mathrm{H} \& \mathrm{E}, \times 200)$. (e) Osteoclastic giant cells lining cystic spaces $(\mathrm{H} \& \mathrm{E}, \times 400)$. (f) Positive immunostaining with CD68 demonstrates the histiocytic origin of the cyst-lining cells $(\times 100)$.

margins and prominent stromal cellularity, with growth imbalance in favor of the stroma (stromal overgrowth), a malignant PT should be considered. The presence of nuclear pleomorphism, numerous mitoses ( $\geq 10$ mitoses/10 HPF), necrosis and heterologous elements in the stroma also support malignancy. The term borderline PT is considered for the cases that do not fit into any of the 2 other groups [1]. In our case, the tumor showed stromal cellularity with increased mitotic activity, atypical mitotic figures, and marked nuclear pleomorphism even in the small tissue fragment obtained by incisional biopsy.

Phyllodes tumors mostly occur in perimenopausal or postmenopausal women with a mean age of 45 years [10]. Most of these tumors are smaller than $5 \mathrm{~cm}$ [11]. The patients usually refer to the clinic with palpable breast lumps and/or mammographic abnormalities detected incidentally. In the physical examination, a wellcircumscribed, firm, mobile mass is noticed. If the tumor is larger than $4 \mathrm{~cm}$ or the patient has any history of rapid growth in a few months, a PT should come to mind, clinically. Malignant PTs may cause nipple retraction or skin ulceration. However, there is no exact clinical clue to differentiate a malignant PT from a benign PT. Axillary lymph nodes are usually not palpable. Our patient referred to the clinic after delivery, with findings reminiscent of a breast abscess. Since the breasts become larger and firmer due to 
the physiological changes during pregnancy and the lactation period, any underlying breast lesions may be obscured. Therefore, a careful breast examination at the first visit of pregnancy is important [9]. Similarly, malignant breast lesions could be mistakenly evaluated as benign and this would delay the correct diagnosis, resulting in more advanced disease [12].

Phyllodes tumors have a tendency for local recurrence, which strongly relates to the status of the surgical margins. The rates of recurrence have been reported as approximately $29 \%$ for borderline and 35\% for malignant PTs [13]. Local excision with a wide surgical margin (at least $1 \mathrm{~cm}$ ) has been accepted as convenient treatment for large-volume breasts and small PTs [14, 15]. Simple mastectomy is an option for cases in which tumor-free margins cannot be provided by local excision [15]. Adjuvant radiotherapy has been recommended for cases with tumors closer than $1 \mathrm{~cm}$ to the margins [14]. Axillary dissection is not performed routinely due to the tendency of PTs to spread via the hematogenous way, similar to sarcomas. Lymphadenectomy is indicated in the presence of clinically suspicious lymph nodes [16]. A simple mastectomy was performed in the current case since the tumor covered the entire breast tissue. Our patient had clear surgical margins but the tumor was closer than $1 \mathrm{~mm}$ to the fascia of the pectoral muscle. Although malignant PTs usually do respect anatomical borders, like a fascia, adjuvant radiotherapy following mastectomy was suggested to our young patient. However, she did not admit. After mastectomy, the rate of local recurrence in the chest wall has been reported as approximately $10 \%$ [13]. Chaney et al. [17] have reported that adjuvant radiotherapy is considered for patients with malignant PTs, after breast-conserving surgery and mastectomy if the tumor size is $>10 \mathrm{~cm}$ or if the margins are positive or the margin distance is less than $5 \mathrm{~mm}$. Similarly, adjuvant radiotherapy is recommended for patients with margin-negative breast-conserving surgery (margin width $<1 \mathrm{~cm}$ ) for local control of borderline and malignant PTs in the study of Barth et al. [13].

Only a few cases of PTs during pregnancy have been reported in the literature between 1998 and 2011 [2-9]. The median age of the patients was 31 years. 1 patient had bilateral tumors. 5 and 3 of 9 tumors were malignant and benign, respectively. There was no exact diagnosis for 1 tumor. The median tumor size was $13.5 \mathrm{~cm}$. The tumor was excised in 4 cases and mastectomy was performed in the others. 4 patients had been operated on during pregnancy whereas 3 patients had surgery after delivery. The time of operation in 1 patient was unknown. In this report, the age of the patient and the size of the tumor were consistent with the literature. The patient was operated on after labor since her symptoms mimicking a breast abscess became clear after delivery.

High concentrations of estrogen, progesterone and prolactin increase the volume of the breast parenchyma due to the growth of ducts and lobular acinar units. Positive staining for hormone receptors in fibroadenomas and PTs has been reported. Since the hormonal alteration may be the cause of rapid growth in PTs during pregnancy, we examined the hormone receptor status in the tumor tissue, immunohistochemically. While the progesterone receptor was weakly positive, the estrogen receptor was negative in our case. However, the status of hormone dependency of PTs is still accepted as uncertain [2].

$\mathrm{ABC}$-like areas are believed to be a reactive lesion rather than neoplastic and defined within some tumors such as chondroblastoma, giant cell tumors, fibrous dysplasia, chondrosarcoma, and gastrointestinal stromal tumors [18, 19]. Some authors claim that $\mathrm{ABC}$-like areas may represent the result of hemodynamic changes in preexisting vascular lesions. Some others indicate that they develop against local hemorrhage. We consider that both the physiologically increased breast vascularization during pregnancy and the tendency of rapid growth of PTs may have caused the formation of $\mathrm{ABC}$-like areas in the current tumor.

In conclusion, the enlargement of the breast volume during pregnancy may complicate a tumor diagnosis in the breast. Therefore, clinical breast examination at the first visit of pregnancy is important. A few cases with PTs during pregnancy have been reported. To the best of our knowledge, this is the first case with $\mathrm{ABC}$-like areas in a newly delivered woman. There is no adequate information in terms of prognosis of PTs in pregnancy, except for their tendency to grow faster. The presence of ABC-like areas may represent the rapid growth of the tumor. Excision with at least 1 $\mathrm{cm}$ of clear margins is recommended for adequate treatment of PTs. The role of adjuvant radiotherapy is still controversial.

\section{Disclosure Statement}

The authors have no conflict of interest to declare.

\section{References}

1 Lakhani SR, Ellis IO, Schnitt SJ, Tan PH, van de Vijver MJ: WHO Classification of Tumors of the Breast, ed 4. Lyon, IARC Press, 2012

2 Way JC, Culham BA: Phyllodes tumour in pregnancy: a case report. Can J Surg 1998;41:407-409.

3 Aranda C, Sotelo M, Torres A, Zárate M: Phyllodes tumor and pregnancy. A report of a case. Ginecol Obstet Mex 2005;73:387-392.

4 Ray S, Basak S, Das S, Pal M, Konar H: Malignant phylloides tumor of breast in a pregnant woman with coincidental nulliparous vaginal prolapse. Iran J Med Sci 2011;36:315-317
5 Mrad K, Driss M, Maalej M, Romdhane KB: Bilateral cystosarcoma phyllodes of the breast: a case report of malignant form with contralateral benign form. Ann Diagn Pathol 2000;4:370-372.

6 Sharma JB, Wadhwa L, Malhotra M, Arora R, Singh S: A case of huge enlargement of cystosarcoma phylloides of breast in pregnancy. Eur J Gynecol Reprod Biol 2004;115:237-239.

7 Simpson SA, Redstone J, Aziz MS, Bernik SF: Large malignant phyllodes tumor with rapid growth during pregnancy: images of a case. Breast J 2007;13:620-621.
8 Nejc D, Pasz-Walczak G, Piekarski J, Pluta P, Bilski A, Sek P, Potemski P, Durczynski A, Wronski K, Jeziorski A: Astonishingly rapid growth of malignant cystosarcoma phyllodes tumor in a pregnant woman - a case report. Int J Gynecol Cancer 2008;18:856-859.

9 Blaker KM, Sahoo S, Schweichler MR, Chagpar AB: Malignant phylloides tumor in pregnancy. Am Surg 2010;76:302-305.

10 Bernstein L, Deapen D, Ross RK: The descriptive epidemiology of malignant cystosarcoma phyllodes tumors of the breast. Cancer 1993;71:3020-3024. 
11 Spitaleri G, Toesca A, Botteri E, Bottigleri L, Rotmensz N, Boselli S, Sangalli C, Catania C, Toffalorio F, Noberasco C, Delmonte A, Luini A, Veronesi P, Colleoni M, Viale G, Zurrida S, Goldhirsch A, Veronesi U, De Pas T: Breast phyllodes tumor: a review of literature and a single center retrospective series analysis. Crit Rev Oncol Hematol 2013;88:427-436.

12 Hogge JP, De Paredes ES, Magnant CM, Lage J: Imaging and management of breast masses during pregnancy and lactation. Breast J 1999;5:272-283.

13 Barth RJ, Wells WA, Mitchell SE, Cole BE: A prospective, multi-institutional study of adjuvant radiotherapy after resection of malignant phyllodes tumors. Ann Surg Oncol 2009;16:2288-2294.
14 Asoglu O, Ugurlu MM, Blanchard K, Grant CS, Reynolds C, Cha SS, Donohue JH: Risk factors for recurrence and death after primary surgical treatment of malignant phyllodes tumors. Ann Surg Oncol 2004;11: 1011-1017.

15 Mituś J, Reinfuss M, Mituś JW, Jakubowicz J, Blecharz P, Wysocki WM, Skotnicki P: Malignant phyllodes tumor of the breast: treatment and prognosis. Breast J 2014;20:639-644.

16 Reinfuss M, Mituś J, Duda K, Ryś J, Smolak K: The treatment and prognosis of patients with phyllodes tumor of the breast: an analysis of 170 cases. Breast J 1996;77:910-916.
17 Chaney AW, Pollack A, McNeese MD, Zagars GK: Adjuvant radiotherapy for phyllodes tumor of breast. Radiat Oncol Investig 1998;6:264-267.

18 Candanedo-Gonzalez F, Camacho-Rebollar L, Uscanga CC, Utrilla AR, Bucio ME, Rodriguez SS, Hernandez LM: Gastrointestinal stromal tumor of the ampulla of Vater with osteoclastic giant cells, osteoidlike matrix deposition, and aneurysmal bone cyst-like features. Ann Diagn Pathol 2013;17:372-376.

9 Do IG, Park CK, Kim KM: Gastrointestinal stromal tumour with osteoclast-like giant cells and aneurysmal bone cyst-like features. Pathology 2009;41:396-397. 